required senior clinical leadership that is committed, visible and facilitative. The support provided by the medical director and trust executive team was crucial to enable the team to experiment and apply the principles of reducing unwarranted variation.

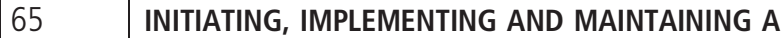 COMPREHENSIVE EDUCATIONAL PROGRAM FOR DONOR CLINICIANS WITHIN NHS BLOOD AND TRANSPLANT}

JB Muller*, L Gaum, S Narayan. NHS Blood and Transplant, UK

\subsection{6/leader-2018-FMLM.63}

Background NHS Blood and Transplant (NHSBT) is responsible for the efficient, safe and reliable supply of blood in England. Within NHSBT, the Clinical Support Team (CST), with Donor Consultants, SAS Doctors and Senior Nurse Practitioners based at different sites, are responsible for the care of blood donors.Continuing professional development (CPD) is vital in this regard. Educational events were however sporadic and not centrally managed. An educational quality improvement project was therefore initiated in 2017. Existing Information Technology (IT) was used to deliver a high quality, diverse, fit-for-purpose and cost-effective educational program.

Method An 'Assess-Plan-Do-Review' framework was followed with an initial 'scoping' meeting to identify the educational needs, promoting a culture of collective ownership. A dedicated teleconference line was allocated and all educational resources were saved in an accessible platform.Sessions were delivered by invited speakers, maximum 1 hour in length, on different days of the week, 4-6 weekly, covering a wide range of topics. A SurveyMonkey questionnaire was circulated in November 2017 to evaluate this initiative.

Results Twenty successful educational sessions have been delivered so far, covering a range of topics. Operational colleagues have also joined in some sessions promoting shared learning and inter-professional education. With an $83 \%$ response rate to the questionnaire, it confirmed that the program's structure was appropriate and addressed educational needs successfully. Suggestions for improvement were captured, including the use of Skype and establishing a Journal Club.

Conclusion It resulted in a positive, collaborative climate and an enthusiastic, participatory learning environment. Reliable IT facilities are vital for successful delivery of such a program to geographically dispersed teams. Commitment and constant review is necessary to ensure the educational program remains fit-for-purpose.

\section{IMPROVING INFORMATION PROVISION IN DELIRIUM CARE}

Myles Potter*, Akinlolu Oluwole-Ojo, Tausif Huq. King's College London, UK

\subsection{6/leader-2018-FMLM.64}

Background Delirium is a common hospital presentation among the elderly population. Chronicity of this condition necessitates that relatives and carers have better access to information. Patient information leaflets offer a simple and cost-effective solution to managing patient education.

Aim(s)/objectives To improve leaflet distribution in Plan, Do, Study, Act (PDSA) cycle 1 and to improve information, aesthetics and educational quality of the current delirium information leaflet in PDSA cycle 2.

Methods PDSA cycle 1 focused on leading improvement in leaflet distribution. Interventions included putting up posters around the ward, introducing the project in team meetings, via emails and moving the leaflet stand to a more visible location. In PDSA cycle 2, baseline data on the quality of the current leaflet was gathered using a questionnaire. A pilot leaflet was developed based on the feedback. The pilot leaflet was surveyed again for quality assessment and to gain further feedback.

Results In PDSA cycle 1, delirium leaflet distribution for weeks 1-3 was 40\%, $0 \%$ and 20\% respectively. Following an implementation of intervention measures, it increased to $110 \%, 120 \%$ and $100 \%$ in weeks $4-6$ respectively. In PDSA cycle 2, mean 'Aesthetic Quality' score improved from 5.14 to $8.71(\mathrm{p}<0.00001)$, 'Quality of Information' from 5.86 to 8.94 $(\mathrm{p}<0.00001)$ and 'User Knowledge Rating' from 5.43 to 9 $(\mathrm{p}=0.00003)$.

Conclusion Overall, we achieved an increase in leaflet distribution and succeeded in developing a new and improved pilot leaflet. Due to the generalisable nature of this quality improvement project, we believe that the framework can be used elsewhere to improve health information management and provision.

\section{Quality improvement}

\section{IMPROVING EFFICIENCY OF CARE FOR ADULT TYPE 2 DIABETIC PATIENTS UNDER SECONDARY CARE}

${ }^{1}$ Naveed Khan*, ${ }^{1}$ Faisel Alam, ${ }^{1}$ Aaron Morjaria, ${ }^{1}$ Michael Walsh, ${ }^{2}$ Yashica Nathan. ${ }^{1}$ GKT School of Medical Education, King's College London; 'Lewisham and Greenwich NHS Trust

\subsection{6/leader-2018-FMLM.65}

This quality improvement paper aimed to improve the efficiency of care for type 2 diabetes mellitus patients. This was done by analysing why patients are not being discharged from hospital diabetic care back to the community whilst creating a novel discharge criterion to help standardise the process. In England over 9\% of the population have been diagnosed with diabetes; with $£ 13$ billion a year being spent on treatment. In the current financial climate, we must ensure that the vast resources being spent are spent as efficiently as possible.

As medical students at the Lewisham and Greenwich trust we found a lack of a discharge criterion made it difficult to discharge patients from hospital care back to the community. Therefore, to improve quality of care and bring cost savings to the department, we aimed to define a clear discharge criterion to de-escalate patients to primary/community care, maintaining optimum care, and increasing the number of discharged patients by $10 \%$ over a 12 month period.

We performed two PDSA cycles on 501 randomly selected patients and found an extra $11.27 \%$ of patients could be deescalated down to community care (from secondary) using our criteria. This quality improvement project could optimise and 
improve quality of care for diabetic patients in the appropriate setting.

Diabetes care is estimated to account for at least 5\% of UK total healthcare expenditure, and up to $10 \%$ of NHS expenditure. Specifically, each outpatient appointment costs on average $£ 115$., thus reducing inappropriate patient loads provides considerable cost savings for the trust. This model could be translated across to other departments and other trusts to optimise and improve the quality of care for many thousands of patients.

\section{Developing effective leaders}

\section{THIS PROGRAME WAS DELIVERED THROUGH THE GSTT SCHOOL OF IMPROVEMENT (POSTGRADUATE MEDICAL EDUCATION) IN A LONDON TEACHING HOSPITAL WITH A LARGE COHORT OF FOUNDATION AND SPECIALIST TRAINEE DOCTORS. THE MOST RECENT NATIONAL TRAINEE SURVEY 2018 HAS DOCUMENTED}

${ }^{1}$ Daghni Rajasingam*, ${ }^{2}$ Anna Cheshire, ${ }^{2}$ David Peters. 'Guys and St Thomas' Hospita Foundation Trust; ${ }^{2}$ Centre for Resilience, University of Westminster

\subsection{6/leader-2018-FMLM.66}

This programme was delivered through the GSTT School of Improvement (Postgraduate Medical Education) in a London teaching hospital with a large cohort of foundation and specialist trainee doctors. The most recent national trainee survey 2018 has documented that significant numbers of doctors in training are burnt out and stressed by their clinical work. This impacts on the quality and safety of care provided in an already 'stressed NHS'. Resilience workshops were delivered as part of mandatory training (through a bespoke, evidence based workshop) for all foundation year doctors and the trust's junior doctor leadership group.Follow up with qualitative questionnaire occurred after the workshop.

Questionnaires were used to collect quantitative and qualitative data from participants at three time points: immediately prior the workshop (baseline), immediately after the workshop (post workshop), and two months after the workshop (followup). Outcome measures collected included perceived stress and positive well-being scales.

The participants reported high levels of stress at baseline with comparisons between baseline and 2 month follow-up questionnaires revealing improved stress levels and well-being ratings. $81 \%$ of participants felt that that topics covered were useful for their work and over three-quarters of participant said that they intended to use some of the techniques they had learnt on the workshop. $76 \%$ of those who completed a follow-up questionnaire had actually done something differently.

This workshop which covered the neurobiology of resilience, the performance curve and a resilience inventory was particularly suited to addressing the needs of doctors who are familiar with the science behind evidence based interventions. This systemic approach to addressing resilience across the workforce needs to be an integrated generic component of postgraduate medical training if we are to future proof the NHS to continue delivering high quality, safe care to patients.

\section{Leading innovation and improvement}

\section{OPERATING THEATRE QUALITY IMPROVEMENT FOR SUSTAINABLE INCREASES IN PATIENT SAFETY AND EFFICIENCY: A REALIST REVIEW}

${ }^{1}$ Aditya Kale*, ${ }^{2}$ Ross Millar. ${ }^{1}$ University of Birmingham, College of Medical and Dental Sciences; ${ }^{2}$ Health Services Management Centre

\subsection{6/leader-2018-FMLM.67}

Introduction The ever-increasing demand for NHS services alongside heavy financial pressures makes the focus on healthcare quality more important than ever before. Patient safety and efficiency are key domains of quality improvement (QI), especially in the operating theatre setting. Operating theatres, being the costliest departments in hospitals are an ideal focus for efficiency gains. The current financial climate of the NHS necessitates a focus on efficiency, whilst also keeping patient safety at the centre of attention.

Aim This review aims to address the question of sustainability in quality improvement, focusing on the two closely linked domains of patient safety and efficiency in the operating theatre setting.

Method This study was a realist review of qualitative and quantitative evidence. Data saturation was reached at 26 papers, however a further 5 studies were fully analysed to confirm this. 8 studies focused on non-technical skills training, 6 focused on checklist implementation, 8 considered process or workflow redesign, and 9 studies relied on other methods. It must be noted however that although interventions are grouped as such, there is significant variability within, and overlap between groups, with some interventions being more complex and incorporating multiple tools as part of the same intervention.

Conclusion The two main concepts to consider refined throughout the review are as follows:

a. A culture of continuous, data-driven quality improvement considering staff needs and perceptions, with support from all levels of an institution, can produce sustained increases in both efficiency and patient safety. Process integration is a vital component of sustainable QI in all stages of planning and implementation.

b. Quality improvement strategies can target patient safety and efficiency in combination, without significant compromise. Consideration of both domains can help align the goals of different stakeholders, enhancing positive staff perceptions and thus participation, resulting in sustained uptake.

\section{An integrated leadership training programme}

\section{TOMORROWS LEADERS, MAXIMISING POTENTIAL - AN INTEGRATED LEADERSHIP TRAINING PROGRAMME}

Daghni Rajasingam, Adam Jakes, Claire Mallinson*. Guys and St Thomas NHS Foundation Trust

\subsection{6/leader-2018-FMLM.68}

The junior doctor leadership group (JDLG) was developed within a London teaching hospital for a cohort of junior doctors and local management trainees. This leadership 